\title{
Effective size control through solvents in microwave aided synthesis of GSH- CdTe quantum dots
}

\author{
P.Bichpuria ${ }^{1 *}$, A.Oudhia $^{2}$ \\ 1,2 (Dept. Of Physics, Govt. V.Y.T. PG Autonomous college, Durg (C.G.), India)
}

\begin{abstract}
Highly luminescent GSH capped CdTe quantum dots (GSH-CdTe QDs) were prepared through a facile microwave $(M W)$ aided wet chemical method. The mechanism of effective size control through the solvents is proposed. The Small angle X-ray scattering (SAXS) measurements show that the size dispersion of QDs can be precisely controlled through the selection of appropriate solvents. The high boiling point and high loss tangents were found to be effective in controlling the size dispersion in MW aided synthesis. The PL studies show that the sharpness of the emission peaks depends on the size dispersion.
\end{abstract}

Keyword: GSH(Glutathione),Luminescent, Microwave (MW), Small angle X-ray scattering (SAXS), Quantum dots.

\section{Introduction}

QDs are being intensively used for producing solar panels [1,2] optoelectronic devices [3] and as fluorescent probes in bio-imaging and bio-sensing [4-6] among other applications. QDs have many advantages such as sharp emission spectra, higher chemical stability, tunable optical properties, photochemical stability and high quantum yields as compared to organic fluorescent material. These properties have broadened the range of applications of QDs in advance studies like protein trafficking [7] and in the treatment of cancer [8-10].

Synthesis of QDs through high temperature thermal decomposition of organo-metallic compounds in high boiling point (B.P.) toxic organic solvents needs long reaction time and involves complex synthesis procedures producing QDs with poor biocompatibility [10-16]. So there is a quest for new, simpler and efficient synthetic routes with alternative solvents. Present study focuses on synthesis of size controlled CdTe QDs through a fast and facile MW [17] aided wet chemical route. Glutathione (GSH)-capped CdTe QDs (GSH-CdTe) were synthesized under mild conditions. These GSH-CdTe QDs show similar optical properties as QDs synthesized using more complicated and expensive methods. MW irradiation is used as an efficient heating source which creates numerous nucleation sites in the solution, leading to the formation of homogeneous and size controlled nanoparticles [18]. Synthesis of ZnS QDs using different polar solvents with high MW absorption is already reported [19].Moreover, the quantum confinement effects in QDs are size dependent and are instrumental in determining their optical properties, so the solvents were chosen carefully to get a better control over the particle size distribution of CdTe QDs. A mechanism to describe the role of solvents in controlling the size dispersion is proposed for a better understanding of the process.

\section{Experimental}

Present study describes a simple protocol for the aqueous synthesis of CdTe QDs using $\mathrm{CdCl}_{2}, \mathrm{~K}_{2} \mathrm{TeO}_{3}$ and GSH.CdCl 2 and $\mathrm{K}_{2} \mathrm{TeO}_{3}$ were used as the $\mathrm{Cd}^{2+}$ and $\mathrm{Te}^{2-}$ sources. The interaction between these two compounds with microorganisms is well studied[20-23].Further, a biological thiol and a tri-peptide GSH works both as a reducing and capping agent for the aqueous synthesis of CdTe QDs[24], found in abundance in cells and are suitable for the stabilization of CdSe and CdTe QDs[25,26]. Moreover, all of the operations could be performed in air avoiding the need for inert atmosphere due to the fact that $\mathrm{K}_{2} \mathrm{TeO}_{3}$ is air stable.

The synthesis of CdTe QDs and their subsequent coating were as follows-

$182 \mathrm{mg} \mathrm{CdCl} 2.2 .5 \mathrm{H}_{2} \mathrm{O}$ was diluted with $40 \mathrm{ml}$ of Ethylene glycol (EG). During the constant stirring $240 \mathrm{mg}$ GSH,10 mg Sodium citrate, $2 \mathrm{ml}$ of $\mathrm{K}_{2} \mathrm{TeO}_{3}$ sol and $20 \mathrm{mg}$ of $\mathrm{NaBH}_{4}$ were added into Cadmium sol. $1 \mathrm{M} \mathrm{NaOH}$ was then used to adjust the $\mathrm{pH} 10$ under vigorous stirring. The mixture was kept in $\mathrm{MW}$ for $1 \mathrm{~min}$ at $300 \mathrm{~W}$. The mixture was cooled down to $\sim 50^{\circ} \mathrm{C}$. The as prepared CdTe solution concentrated to $1 / 4{ }^{\mathrm{Th}}$ of the original volume was precipitated using 2 propanol and collected via centrifugation. CdTe QDs dispersion was prepared by re-dissolving this colloid precipitate in $3 \mathrm{ml}$ DD water. A similar procedure was followed for preparing CdTe QDs in two different high B.P.organic solvents namely HPLC water and Toluene. 


\section{Results And Discussions}

\subsection{Selection of high MW absorbing solvents}

In present study MW irradiations were used as the source of energy and the high B.P. solvents were chosen for synthesis as they are highly MW absorbing. MW based methods have an edge over the other methods described above as they can be scaled up for large scale production without suffering unwanted thermal gradient effects[27].Selection of precursors and solvent play a very important role in MW-assisted reaction kinetics. Table 1 shows that EG had the highest B.P. as well as remarkably high value of the loss tangent, both affect the heating process, which finally led to the formation of QDs with minimum size dispersion. At the higher temperatures allowed by organo-metallic synthesis, reaction rates are faster and crystallinity is generally higher. Furthermore, there is more thermal energy to help each add-atom find more energetically favorable bonding positions in the crystal lattice, thus reducing defects by annealing during growth. Based on these findings, EG was chosen as solvent for all further optical studies.

Table 1 Comparison of properties of organic solvents used to synthesize CdTe QDs

\begin{tabular}{|l|l|l|l|l|}
\hline \multirow{2}{*}{ S.No } & \multirow{2}{*}{ Properties of organic solvents } & \multicolumn{2}{l|}{ Organic solvents } \\
\cline { 3 - 5 } & & Toluene & EG & HPLC water \\
\hline 1. & B.P. $\left({ }^{\circ} \mathrm{C}\right)$ & 110 & 197.3 & 100 \\
\hline 2. & Density $\left(\mathrm{kg} / \mathrm{m}^{3}\right)$ & 867 & 1110 & 1000 \\
\hline 3. & Dielectric constant & 2.38 & 37 & 80.10 \\
\hline 4. & Loss tangent $(\tan \delta)$ & 0.04 & 1.35 & 0.16 \\
\hline
\end{tabular}

The particle size distribution was determined by SAXS studies shown in Fig. 1 and the related data is compiled in Table 2.
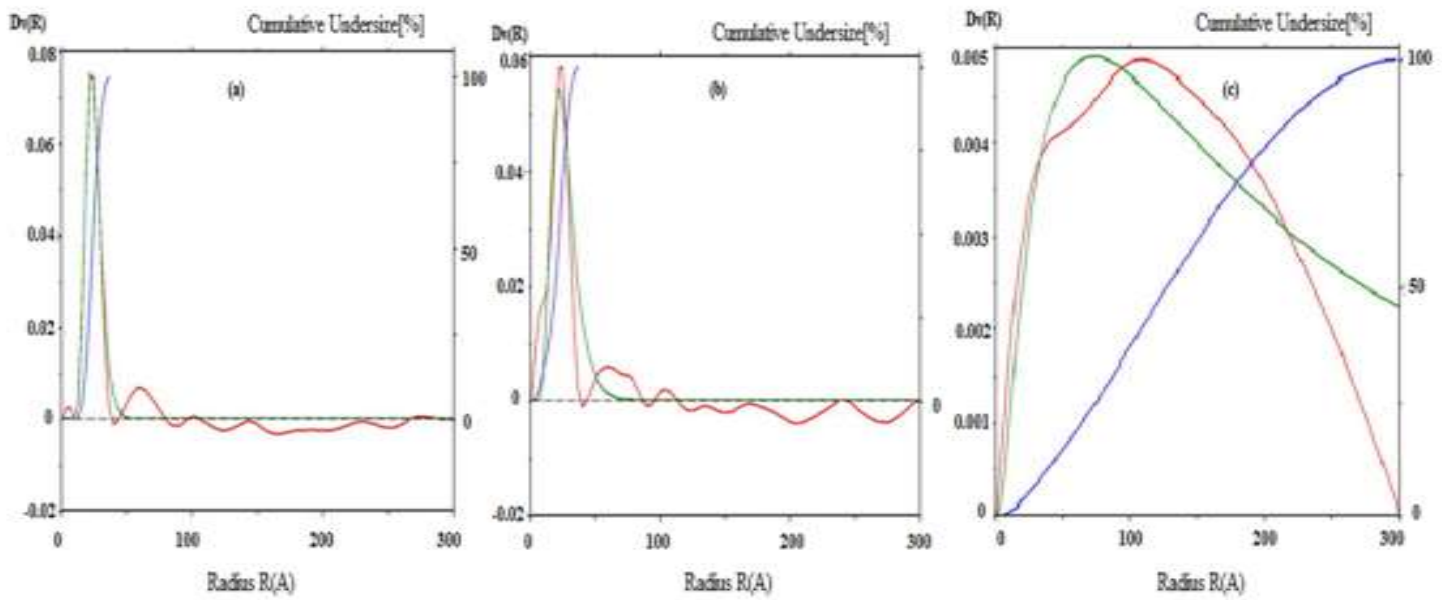

Fig. 1 SAXS studies showing size distribution of GSH-CdTe nanoparticles by volume using (a) EG (b) Toluene (c) HPLC water as solvents.

The standard deviation is minimum in the case of EG, which has the highest boiling point.

Table 2 Details of SAXS studies of CdTe particles obtained using various solvents

\begin{tabular}{|l|l|l|l|l|l|l|}
\hline S. no. & Sample name & Organic solvents & $\begin{array}{l}\text { Absorption factor } \\
\text { of samples }\end{array}$ & $\begin{array}{l}\text { Average radius } \\
(\mathrm{nm})\end{array}$ & $\begin{array}{l}\text { Standard } \\
\text { deviation } \\
(\%)\end{array}$ & $\begin{array}{l}\text { Surface } \\
\text { volume } \\
\text { ratio(S/V) }\end{array}$ \\
\hline 1. & GSH-CdTe QDs & Toluene & 1.51 & 2.07 & 34.25 & $0.181 / \mathrm{A}$ \\
\hline 2. & GSH-CdTe QDs & EG & 1.61 & 2.33 & 20.51 & $0.131 / \mathrm{A}$ \\
\hline 3. & GSH-CdTe QDs & HPLC water & 1.98 & 13.06 & 53.24 & $0.041 / \mathrm{A}$ \\
\hline
\end{tabular}

It can be further added that the non polar reaction medium involving dielectric heating mechanism (EG and Toluene) resulted in enhanced reaction rates, higher reaction yield and a better control over the size and hence the optical properties of QDs than polar solvent (HPLC water) showing ionic conductivity mechanism. Further, the dielectric heating heats the whole volume uniformly and reduces thermal gradient effects, when compared to convective heating; allowing a better control over size dispersion [19]. It has been reported that the EG molecules participate in the stabilization of the nanocrystals and passivation of surface defects, with an optimum concentration for production of stably dispersed CdTe [28].

\subsection{Morphological studies}

The SEM micrograph (JEOL EO JSM 5600) shown in Fig.2(a) shows representing GSH-CdTe QDs synthesized using EG as a solvent at magnification 20kv x 10000.This micrograph shows density populated colony of CdTe QDs with almost spherical morphology. The inset attached with Fig. 2 (a) shows that this QDs are well dispersed.The XRD pattern matches with the JCPDS file JCDS card no.65-1046 of wurtzite CdTe (Fig. 2(b)) . 

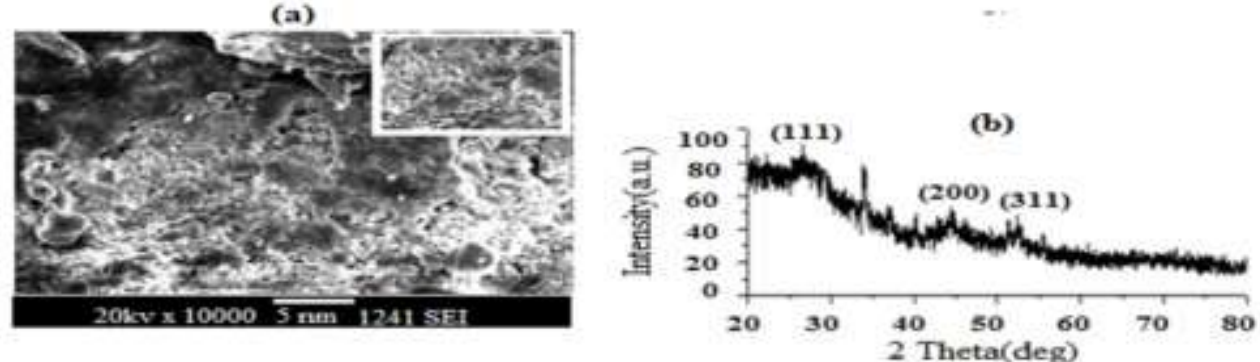

Fig.2 (a) SEM micrograph of GSH-CdTe QDs synthesized using EG as solvent (b) XRD of GSH-CdTe QDs using EG as solvent

However it was interesting to note that the percentage yield and minimum particle size was obtained in case of toluene mediated process. Fig. 3 (a), 3(b) and 3(c) compares the percentage yield obtained for all three solvents used, showing toluene mediated synthesis giving highest $(95 \%)$ yield.

EG-35\% Yield HPLC water- $24 \%$ Yield Toluene- $95 \%$ yield
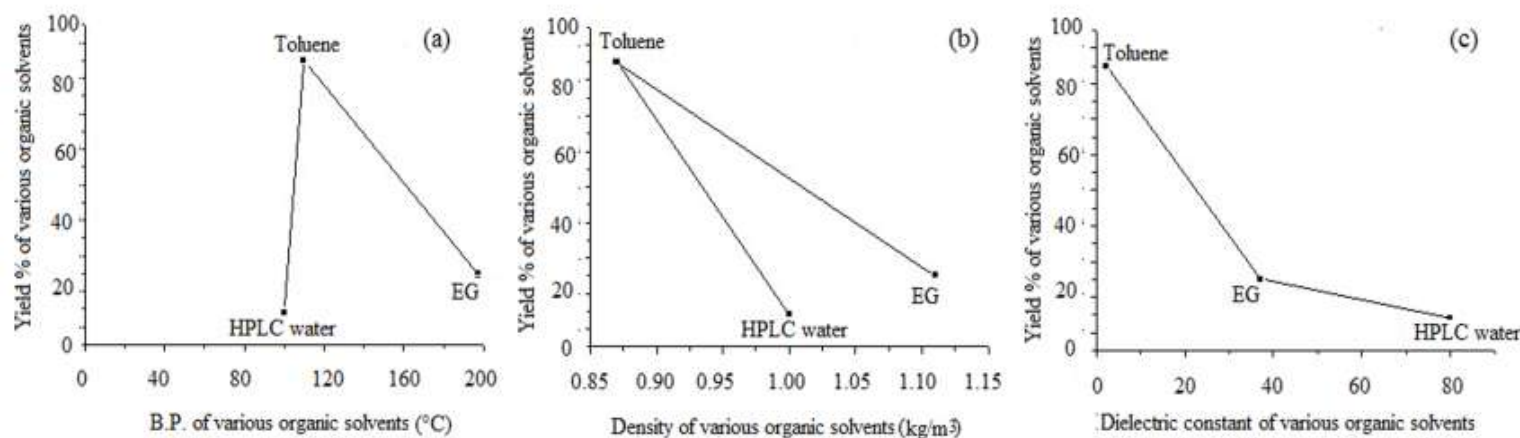

Fig . 3 Percentage yield obtained as a function of (a) B.P. of the solvents used (b) density of the solvents used (c) dielectric constants of the solvents used.

Finally, the MW power and time of irradiation were optimized for toluene for getting higher percentage yield and the values were selected from the graphs shown in Fig.4 (a) and (b).
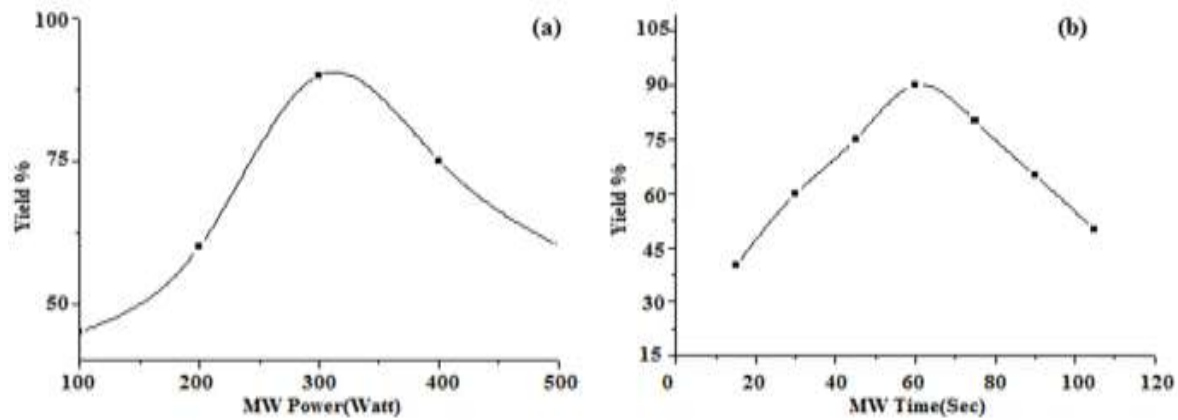

Fig.4 (a) \% yield Vs MW power showing a peak around power $300 \mathrm{~W}$ (b) \% yield Vs MW irradiation time showing max yield at around $60 \mathrm{sec}$.

\subsection{Effective capping mechanism of capping and surface passivation by EG}

There are various strategies developed for getting a narrower size distribution of nanoparticles like to minimize coagulation by adding surfactants to improve dispersion and cause them to repel each other by columbic forces or steric hindrance $[29,30]$. In present case GSH dissolved in various polar/ non-polar solvents was used for capping and surface passivation. It was observed that the nanocrystal size variations are reduced in case of non-polar organic solvents where ligands controlled the growth rate of particles. In wet chemistry routes, the synthesis temperature is limited by the B.P. of the solvent. Growing at lower temperatures produces QDs with higher defect concentrations, which translates into a low photoluminescence quantum yield (PLQY). Present study addresses these problems by adopting MW aided synthetic strategies using high B.P. nontoxic solvents, to achieve a lower particle size distribution along with a sharp PL emission free of peaks related to defects. Apart from this the organic ligands naturally provide surface passivation through binding to the surface during synthesis, which protects the surface from oxidation and minimizes the electronic trapping properties of 
surface defects [30]. It will be interesting to relate the functional groups present in the solvents with the surface passivation mechanism prevailing in them.Fig.5 shows the chemical structures of all three solvents used in the present study.

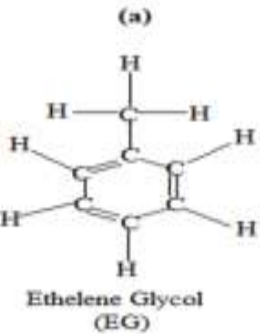<smiles>OCCO</smiles>

Toluene

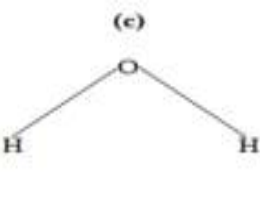

HPL.C Water

Fig. 5 Structures of organic solvents used in the present study (a) EG (b) Toluene (c) HPCL water.

One can attribute the effective passivation of QDs by EG to the methyl group attached to the benzene ring present in them, which has a greater affinity to bind CdTe than the two - OH groups found in toluene attached to a straight carbon chain [31]. Further, the water molecules have a single $-\mathrm{OH}$ ion, whereas at least two water molecules are needed to bind to one $\mathrm{Cd}^{2+}$ ions, this will leave unsaturated bonds resulting in a poor surface passivation as compared to toluene, which has two $-\mathrm{OH}$ ions attached to it. Both (SAXS) (see in Fig.1) and optical studies show a stronger passivation by EG as compared to toluene and water resulting in a better size control.

\subsection{Effect of solvents on the optical properties of QDs}

Fig.6(I) shows the UV-Vis optical absorption spectra of CdTe QDs prepared in various solvents. The sudden increase in absorption indicates direct band to band absorption, depicting the absorption edge. It shows a blue shift with the decreasing particle size. Moreover the sharp absorption edge can be attributed to absence of defect states in the band gap region. Fig.(6)II shows Energy band model showing the defects in bulk CdTe.Cd vacancy, $\mathrm{V}_{\mathrm{cd}}$ gives rise to shallow acceptor states, while $\mathrm{Cd}$ substitution on a Te site, $\mathrm{Cd}_{\mathrm{Te}}$ gives rise to shallow acceptor state. Interstitial $\mathrm{Cd}\left(\mathrm{Cd}_{\mathrm{i}}\right)$ gives rise to a relatively shallow donor state, while Interstitials $\mathrm{Te}\left(\mathrm{Te}_{\mathrm{i}}\right)$ gives rise to deep states [ 32]. However, the defect related hump observed in between 400-500 nm in Fig.6(I) is visible in case of toluene and HPLC mediated QDs, whereas it is absent in the absorption spectra of EG mediated QDs. This again supports the defect free synthesis mechanism of high boiling point organic solvents as EG.
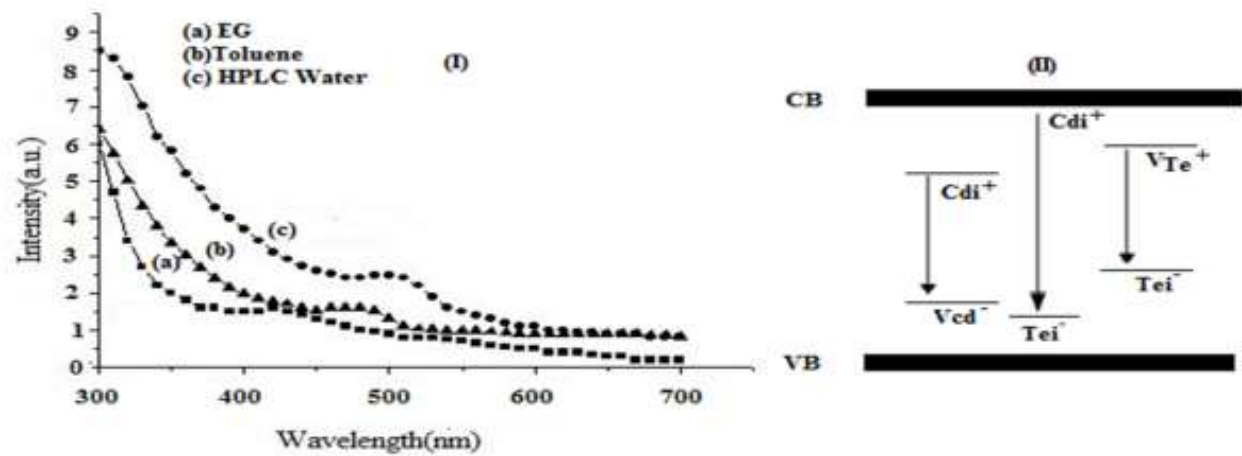

Fig.6 (I) UV-optical absorption of GSH-CdTe QDs using various organic solvents (II) Energy band model defects in bulk CdTe.

The PL emission spectra of the CdTe QDs obtained using various solvents is shown in Fig. 7 it can be observed that the narrowest and sharpest peak is observed in case QDs synthesized in EG. This can be closely related to the narrowest size distribution obtained in this case along with the defect free synthesis mechanism mediated by EG as discussed earlier.

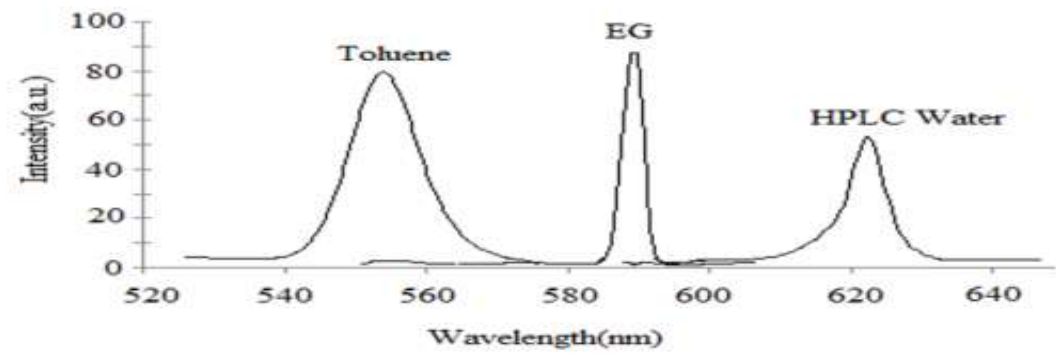

Fig.7 PL of GSH-CdTe QDs using various organic solvents. 


\section{Conclusions}

Present study can be used to achieve effective control over particle size distribution of QDs for applications within the quantum confinement regime, where size controls their physical properties. It was observed that the organic solvents were more effective in controlling the particle size distribution. The boiling point and loss tangent of the solvents were found to be crucial in size controlled MW aided synthesis. SEM micrograph shows well dispersed density populated colony of GSH-CdTe QDs with almost spherical morphology. The sharp and narrow PL emission spectra show the effective size control in EG mediated CdTe QDs.

\section{Acknowledgements}

We acknowledge the kind help and support of Prof S. J. Dhoble at RTM University, Nagpur (Maharashtra) for PL studies, Dr. D.P. Bisen at Pt. Ravishankar Shukla University Raipur(C.G.) for UV-Visible studies, Dr. M. Chopkar asst.prof. and Mr.Suresh Dua, Senior Laboratory Assistant at NIT, Raipur(C.G.) for SAXS studies and Dr. D.M. Phase at CISR Indore for SEM studies.We are also grateful to Mr. N. kulkarni, TIFR (Mumbai) for XRD studies.

\section{References}

[1]. J.H.Bang, and P.V. Kamat, Quantum dot sensitized solar cells. a tale of two semiconductor nanocrystals: CdSe and CdTe, ACS Nano, 3, 2009, $1467-1476$.

[2]. A. Kongkanand, K.Tvrdy, K.Takechi, M. Kuno, and P.V. Kamat, Quantum Dot Solar Cells. Tuning Photoresponse through Size and Shape Control of $\mathrm{CdSe}-\mathrm{TiO}_{2}$ Architecture, Journal of the American Chemical Society, 130, 2008, 4007-4015.

[3]. A. Faraon, D. Englund, I. Fushman, and J. Vuckovic, Local quantum dot tuning on photonic crystal chips, Applied Physics Letter, 90,2007, 213110 .

[4]. A.Hoshino, K.Hanaki, K. Suzuki, and K.Yamamoto, Applications of T-lymphoma labeled with fluorescent quantum dots to cell tracing markers in mouse body, Biochemical and Biophysical Research Communications,314, 2004,46-53.

[5]. H.Han, Z.Sheng, and J. Liang, Electrogenerated chemiluminescence from thiol -capped CdTe quantum dots and its sensing application in aqueous solution, Analytica Chimica Acta, 596,2007,73-78.

[6]. A. Hoshino, N. Manabe, K. Fujioka, K. Suzuki, and M. Yasuhara, et al., Use of fluorescent quantum dot bioconjugates for cellular imaging of immune cells, cell organelle labeling, and nanomedicine: surface modification regulates biological function, including cytotoxicity, Journal of Artificial Organs , 10,2007,149-157.

[7]. S. Muro, X.Cui ,C.Gajewski, J.C. Murciano, and V.R. Muzykantov, et al., Slow intracellular trafficking of catalase nanoparticles targeted to ICAM-1 protects endothelial cells from oxidative stress, American journal of physiology. Cell physiology C , 285, 2003,1339-1347.

[8]. H.M.Azzazy, M.M.Mansour, and S.C.Kazmierczak, From diagnostics to therapy:prospects of quantum dots, Clinical Biochemistry,40 ,2007,917927.

[9]. X.Gao, Y.Cui, R.M. Levenson, L.W. Chung, and S. Nie, In vivo cancer targeting and imaging with semiconductor quantum dots, Nature Biotechnology,22, 2004, 969-976.

[10]. S.Q. Chang, Y.D. Dai, B. Kang, W. Han, and L. Mao, et al., UV-enhanced cytotoxicity of thiol-capped CdTe quantum dots in human pancreatic carcinoma cells, Toxicology Letters, 188,2009,104-111.

[11]. H.Bao, E.Wang, S.Dong, One-pot synthesis of CdTe nanocrystals and shape control of luminescent CdTe-cystine nanocomposites, Small, 2,2006, 476-480.

[12]. Y.He, H.Lu, L.Sai, W.Lai, Q.Fan et al., Synthesis of CdTe nanocrystals through program process of microwave irradiation, Journal of Physical Chemistry B ,110,2006, 13352-13356.

[13]. R. Hardman, A toxicologic review of quantum dots: toxicity depends on physicochemical and environmental factors,Environmental Health Perspectives, 114,2006, 165-172.

[14]. J.Lovrić, S.J.Cho,F.M. Winnik, D.Maysinger, Unmodified cadmium telluride quantum dots induce reactive oxygen species formation leading to multiple organelle damage and cell death, Chemistry \& Biology,12, 2005, 1227-1234.

[15]. R.Schneider, The exposure of bacteria to CdTe-core quantum dots: the importance of surface chemistry on cytotoxicity, Nanotechnology, 20, 2009, 225101.

[16]. N.Gaponik, D.V.Talapin,A.L. Rogach, K.Hoppe, E.V.Shevchenko et al., Thiolcapping of CdTe nanocrystals: an alternative to organometallic synthetic routes, The Journal of Physical Chemistry , 106, 2002, 7177-7185.

[17]. A.Oudhia, A.Choudhary; Microwave pulse-assisted wet chemical synthesis of $\mathrm{ZnO}$ nanoparticles with excellent UV emission; Emerging Electronics (ICEE), IEEE 2nd International Conference, 1-4, 2014

[18]. P.Bichpuria, A.Oudhia, MICROWAVE IRRADIATION ROUTE TO SYNTHESIZE GSH CAPPED CdTe QDS, International Journal of advance research in science and engineering, 4, 2015,101-105.

[19]. Robina Shahid, Green Chemical Synthesis of II-VI Semiconductor Quantum Dots,Ph.D Disertation, Royal Institute of Technology (KTH),Ph.D Disertation, Royal Institute of Technology (KTH), Stockholm ,2012.

[20]. J.M.Pérez, I.L.Calderón ,F.A. Arenas , D.E.Fuentes , G.A.Pradenas, et al., Bacterial toxicity of potassium tellurite: unveiling an ancient enigma, PLoS ONE 2: e211,2007.

[21]. T.G. Chasteen , D.E.Fuentes J.C Tantaleán C.C.Vásquez, (2009) Tellurite: history, oxidative stress, and molecular mechanisms of resistance, FEMS Microbiology Reviews, 33,2009 820-832.

[22]. K.Helbig ,C.Bleuel ,G.J. Krauss, D.H.Nies, Glutathione and transition-metal homeostasis in Escherichia coli, Journal of Bacteriology ,190, 2008, 5431-5438.

[23]. R.J.Turner, J.H.Weiner, D.E.Taylor, Tellurite-mediated thiol oxidation in Escherichia coli, Microbiology, 145, 1999, 2549-2557.

[24]. F.Q.Schafer, GR.Buettner, Redox environment of the cell as viewed through the redox state of the glutathione disulfide/glutathione couple. Free Radical Biology and Medicine ,30,2001,1191-1212.

[25]. J.Tian ,R. Liu ,Y. Zhao, Q.Xu ,S. Zhao, Controllable synthesis and cell-imaging studies on CdTe quantum dots together capped by glutathione and thioglycolic acid, Journal of Colloid and Interface Science ,336,2009, 504-509.

[26]. Y.Zheng ,S. Gao , J.Ying ,Synthesis and cell-imaging applications of glutathione capped CdTe quantum dots, Advanced Materials, 19,2007, 376380.

[27]. A.B. Panda, G. Glaspell, and M.S. El-Shall, Microwave Synthesis of Highly Aligned Ultra Narrow Semiconductor Rods and Wires, Journal of the American Chemical Society, 128,2006,2790-2791

[28]. O.Kulakovich, D. Korbutyak, S. Kalytchuk, S. Budzulyak, O. Kapush, L. Trishchuk, S.Vaschenko, V. Stankevich, A. Ramanenka, Influence of conditions for synthesis of CdTe nanocrystals on their photoluminescence properties and plasmon effect, Journal of Applied Spectroscopy, 79,2012,765

[29]. Tadao Sugimoto, Preparation of Monodispersed Colloidal Particles, Advances in Colloid and Interface Science, 28,1987,65-108

[30]. Ombretts Masala and Ram Seshadri, Synthesis Routes for Large Volumes of Nanoparticles, Annual Review of Materials Research,

34,2004, 41-81.

[31]. Toluene-wikipedia, the free encyclopaedia, en.wikipedia.org/wiki/Toluene.

[32]. Brian E. McCandless and James R. Sites, Cadmium Telluride Solar Cells, Chapter14, defect cdte ,Colorado, USA. 